\title{
CHARACTERIZATION OF REFLECTORS AND ABSORBER COATINGS FOR SOLAR CONCENTRATORS
}

\author{
Rahul B.Waghe \\ Department of Mechanical Engineering, Smt.Kashibai Navale College of Engineering, Pune University, Pune
}

\begin{abstract}
Solar energy has great significance specifically for a country like India which is struggling to meet its energy demand. The project includes development of a test chamber for simulating different conditions of weathering and testing of ordinary glass mirrors and solar mirrors to identify the effect of weathering. This project will help in identifying the appropriate reflector and absorber coating for different locations and also in defining the maintenance schedule. This project is an attempt for testing of reflector and absorber coating which will help in material selection by identifying the merit and demerits of each material helpful in defining the replacement or maintenance cycle. New types of selective coatings and effect of different conditions on performance of reflectors and coatings have been discussed in this paper.
\end{abstract}

Key Words-Solar Energy, Test chamber, Reflector, Absorber coating $* * *$

\section{INTRODUCTION}

Solar energy is a very large, inexhaustible bit dilute source of energy. The power from the sun intercepted by the earth is approximately $1.8 \times 10^{11} \mathrm{MW}$ which is many thousands of times larger than the present consumption rate on the earth of all commercial energy sources. Thus, in principle, solar energy could supply all the present and future energy needs of the world on a counting basis. This makes it one of the most promising of the unconventional energy sources. Solar concentrator is a device that allows the collection of sunlight from a large area and focusing it on a smaller receiver or exit. A conceptual representation of a solar concentrator used in harnessing the power from the sun to generate electricity is shown in Figure 1.

The material used to fabricate the concentrator varies depending on the usage. For solar thermal, most of the concentrators are made from mirrors while for the BIPV system, the concentrator is either made of glass or transparent plastic. These materials are far cheaper than the PV material. The cost per unit area of a solar concentrator is therefore much cheaper than the cost per unit area of a PV material. By introducing this concentrator, not only the same amount of energy could be collected from the sun, the total cost of the solar cell could also be reduced.

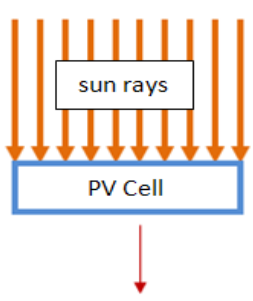

ELECTRICITY

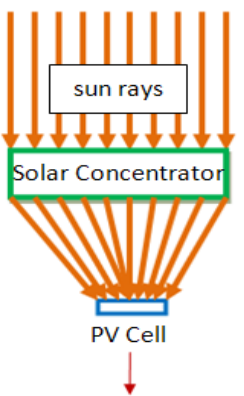

ELECTRICITY
Fig -1: Generating electricity from the sun, with and without a solar concentrator.

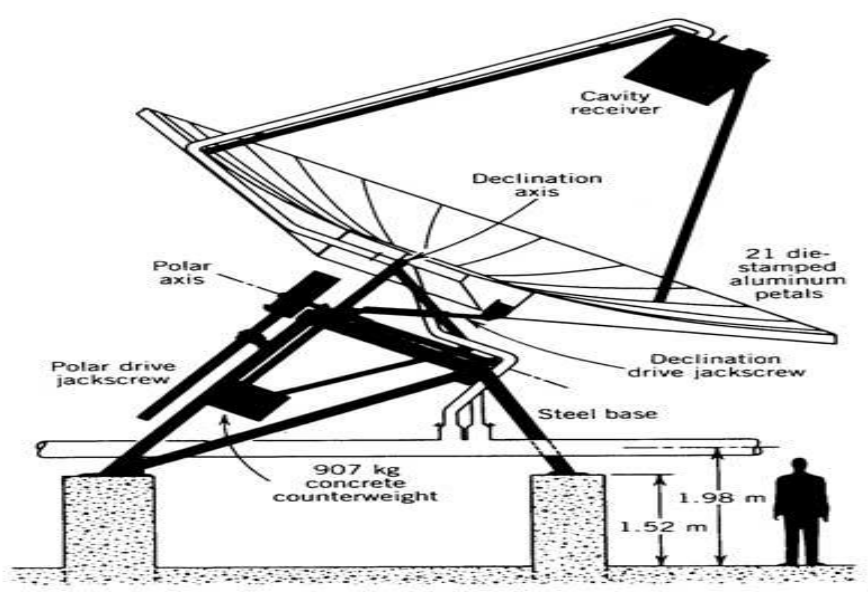

Fig -2: Solar Concentrator 


\subsection{Types of Solar Concentrator}

1. Parabolic Concentrator

2. Hyperboloid Concentrator

3. Fresnel Lens Concentrator

4. Compound Parabolic Concentrator (CPC)

5. Dielectric Totally Internally Reflecting Concentrator (DTIRC)

6. Flat High Concentration Devices

7. Quantum Dot Concentrator (QDC)

\section{DESIGN OF ACCELERATED WEATHERING}

\section{CHAMBER.}

\subsection{Design of Chamber}
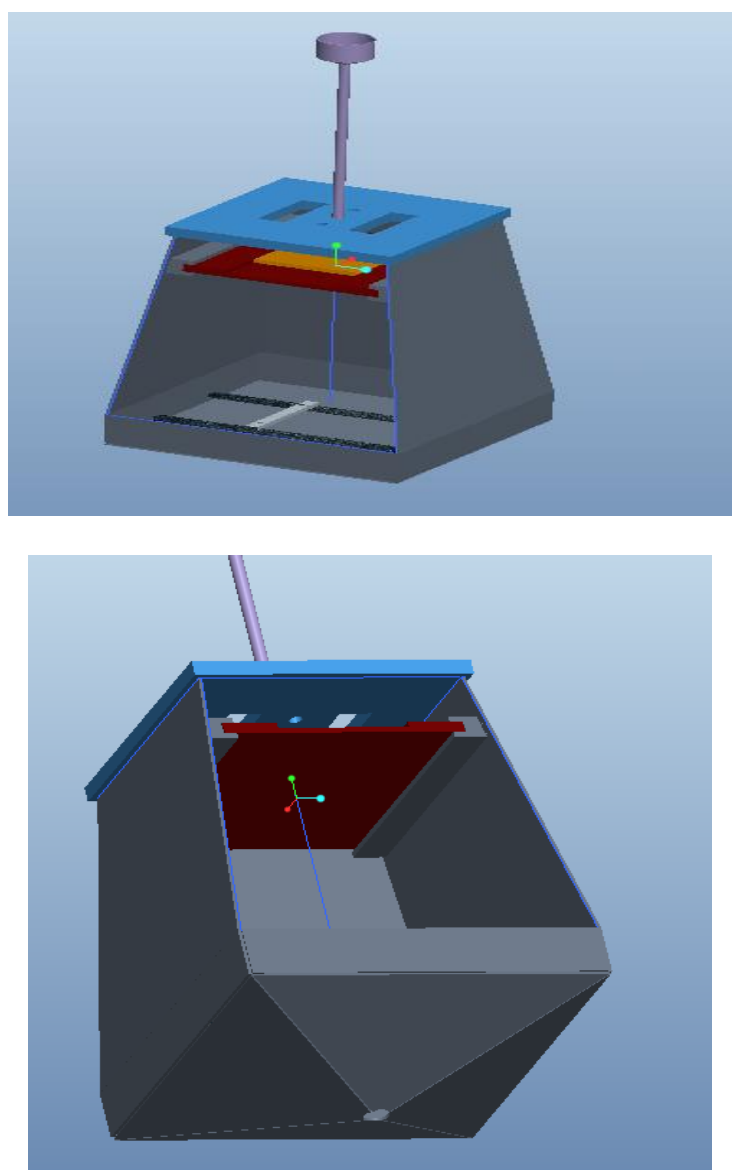

Fig -3: Pro-E Design of accelerated weathering chamber

\subsubsection{Features}

\subsubsection{Cover plate}

1) The cover plate consists of the heating and spraying arrangement with two heaters and water spray nozzles and one sand spray nozzle.

2) The cover plate is made up of insulating material with slots for the heating and spraying elements.

\subsubsection{Heating Element and Sprays}

1) The heating element is a finned air heater as it provides required heating.

2) The temperature of the heater can be controlled by using a thermostat.

3) The size of the finned heater: 160x60 mm.

4) Diameter of the holes provided: $20 \mathrm{~mm}$ and length greater than the heater thickness.

5) Metal nozzles (usually mild steel) are provided for both the sprays. The length of the nozzles is more than the thickness of the heating element.

\subsubsection{Chamber}

1) The walls of the chamber are insulated from the inside for creating ambient conditions.

2) The chamber is given slope such that the bottom square portion is larger than the upper portion.

3) A glass cover is provided as the opening for the chamber.

\subsubsection{Drain}

1) Drain hole for the exit of water and also contains a concentric pipe entering through it for the cooling of the rear side of the plate.

\section{Advantages:}

1) The nozzles are arranged such that the central portion of the plate is affected which is usually favoured while further testing of the test plate.

2) The top cover plate is made up of insulating material for safety.

3) The length of the nozzle is longer such that the water or the sand is not sprayed on the heating element.

4) A thermostat is provided for regulating the temperature of the heating element.

\subsection{Specifications}

\subsubsection{Heater:}

Type: Air Heater (Coil wire in Ceramic)

Voltage: $230 \mathrm{~V}$

Power: 500W

Temperature Range: Up to $200^{\circ} \mathrm{C}$

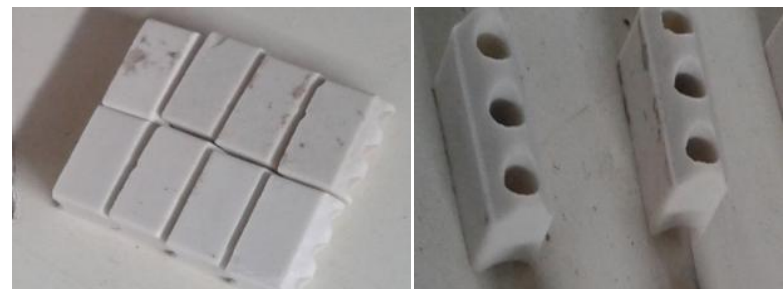

Fig -4: Heater images 


\subsubsection{Nozzles: Water Nozzle (Brass)}

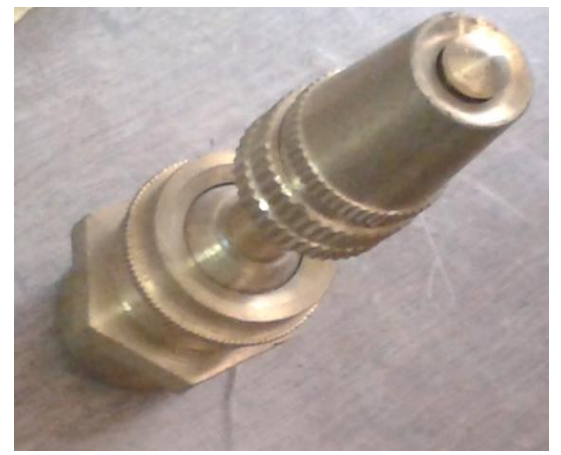

Fig -5: Water nozzle

\subsection{Fabrication}

\subsubsection{Required Raw Material}

Mild Steel Sheet-6ft x 4ft

Thickness-3mm

\subsubsection{Fabrication of the Chamber}

The setup for the weathering chamber was manufactured at SKNCOE workshop. The workshop staff helped in some of the complex operations.

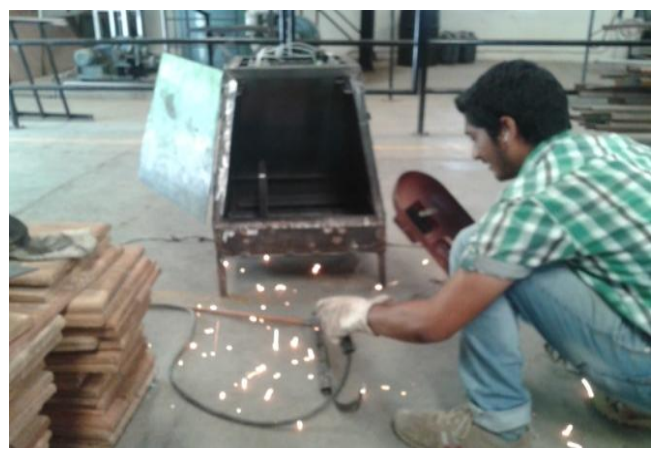

Fig -6: Student performing welding operation

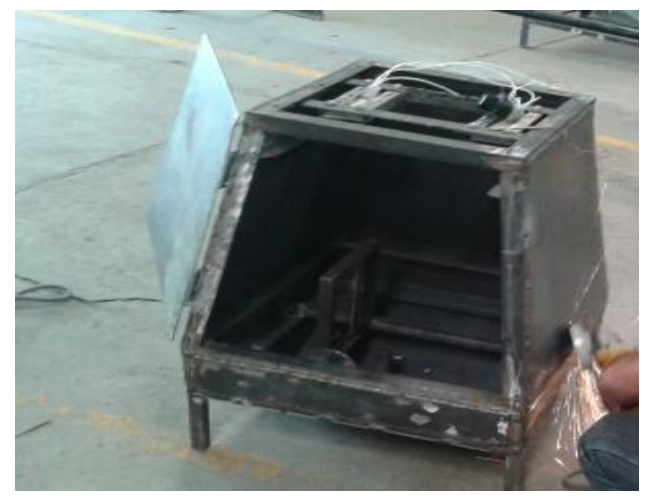

Fig -7: Fabricating the chamber

\section{REFLECTIVITY MEASUREMENT METHODS}

\subsection{Pyranometer}

A Pyranometer is a type of actinometer used to measure broadband solar irradiance on a planar surface and is a sensor that is designed to measure the solar radiation flux density (in watts per meter square) from a field of view of 180 degrees. The solar radiation spectrum extends approximately from 300 to $2,800 \mathrm{~nm}$. Pyranometer usually cover that spectrum with a spectral sensitivity that is as "flat" as possible.

\section{Advantages:}

1) Portable and inexpensive.

2) Applies equally well curved surfaces.

3) Low temperature response, which is an advantage when working under extreme climate conditions.

4) Low non-linearity.

\section{Restrictions in measurement:}

1) The sky must be clear, particularly around the sun.

2) The spectral distribution of incident solar irradiance (Ii) and the incidence angle $(\theta)$ of the solar beam both vary with hour of day and day of year.

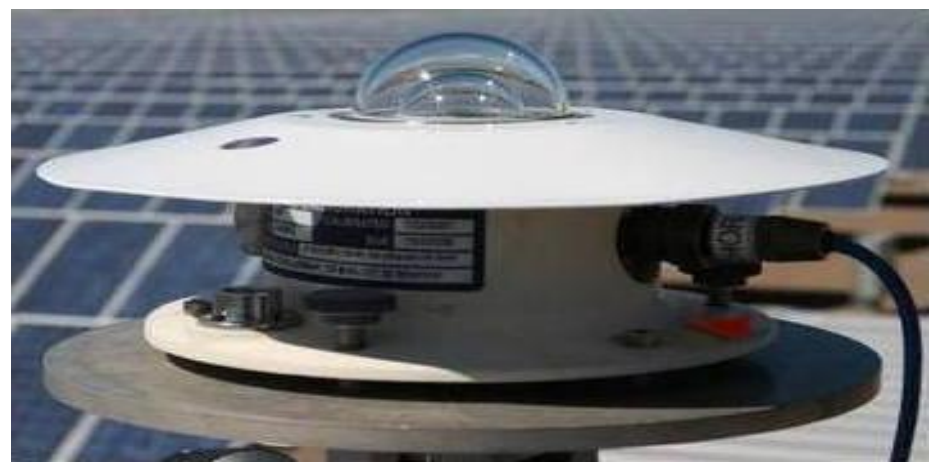

Fig -8: A Pyranometer

\section{ABSORBER COATINGS}

\subsection{Sparc Coating}

1) SPARC is an anti-reflective coating especially developed for solar applications.

2) SPARC is a single-side coating deposited on extra clear patterned glasses Solite and Solatex during the tempering process.

3) Being single-sided, SPARC minimizes the reflection at the glass/air interface without affecting the excellent adhesion between the glass and the interlayer used in laminating photovoltaic modules.

1) Transmission increase (\%): Up to $2.4 \%$ At normal Incidence

2) Energy output increase (\%): Up to 5\% In actual Conditions (kWh) 
4) Tests Cleared: i) Damped Heat ii) Thermal Cycle iii) Climatic SO2 iv) Salt Spray

\subsection{Sun Mirox $T$}

1) SUN MIROXTM THIN is an extra clear extra thin high reflectivity mirror perfectly suited for lamination purposes and use in parabolic dish, parabolic trough collectors, CSP or CPV.

2) Once laminated with an appropriate adhesive onto a support material the mirror is perfectly well protected and shows a very high chemical and mechanical durability.

3) In order to minimize its environmental impact before, during and after service, SUN MIROXTM THIN is copper-free and lead-free.

\subsubsection{Thermal Characteristics}

1) Hemispherical emissivity: 0.84 (Between $-18^{\circ} \mathrm{C}$ and $66^{\circ} \mathrm{C}$ )

2) Expansion coefficient: 9 (EN572between $20^{\circ} \mathrm{C}$ and $300^{\circ} \mathrm{C}$ )

3) Specific heat $(\mathrm{J} / \mathrm{kg} / \mathrm{K}): 720$

4) Thermal conductivity $(\mathrm{W} / \mathrm{m} / \mathrm{K}): 1$

5) Softening point $\left({ }^{\circ} \mathrm{C}\right): 722$

6) Annealing point $\left({ }^{\circ} \mathrm{C}\right): 552$

7) Strain point $\left({ }^{\circ} \mathrm{C}\right): 500$

\subsection{Organic Coatings}

1) Pencil hardness: $3 \mathrm{H}$ to $5 \mathrm{H}$

2) Solvent resistance: $100+$ acetone rubs w/o effect

3) Tape adhesion: no loss after 30 minutes boiling water

\subsubsection{Advantages:}

1) New Technology

2) Low Temperature

3) Heavy Metal Free

\subsection{Almeco Tinox Solar Coatings}

1) TiNOX is an energy trap.

2) Highly selective blue TiNOX energy absorber coatings take up a lot of energy -- some $\mathbf{9 5 \%}$ of incident solar radiation -- and convert it into heat energy.

3) Conventional absorbers lose a large part of that energy as heat radiation. In contrast, TiNOX energy coatings have an extremely low thermal emissivity of less than $4 \%$ in the infrared range to prevent such heat losses.

4) Once the energy has been converted into heat, it stays trapped within the TiNOX absorber.

5) The high performance of the absorber layer therefore requires the greatest possible degree of absorption within the solar radiation range and the lowest possible degree of emissivity in the heat radiation range.

6) TiNOX energy fulfils this requirement ideally:

\section{Solar absorption $\alpha=95 \%$}

Thermal emissivity $\varepsilon<4 \%$

\section{ACCELERATED CHAMBER TESTING}

\subsection{Sand Abrasion Testing}

1) In this test sand is sprayed in ppm on the surface of the plate with the help of pump.

2) Different types of sand were used since plate can be used at any location in the world.

3) In our case the sand is made to fall vertically downwards from a particular height of $1.5-2 \mathrm{~m}$.

\subsubsection{Results}

1) Damage caused by windblown sand is proportional to the particle momentum.

2) Cumulative damage to surfaces is proportional to the sum of damage caused at certain wind velocities multiplied by the infliction time.

3) Properties influenced by sand damage:

i) Absorptivity $\alpha$, Reflection $\rho$, transmission $\tau$

ii) Roughness $R_{a}, R_{z}$, diffusion $d$

iii) Wetting/contact angle $\alpha$, dirt-repellent

iv) Pitting has a strong influence on the wettability of the surface and thus on dirt accumulation and washing processes.

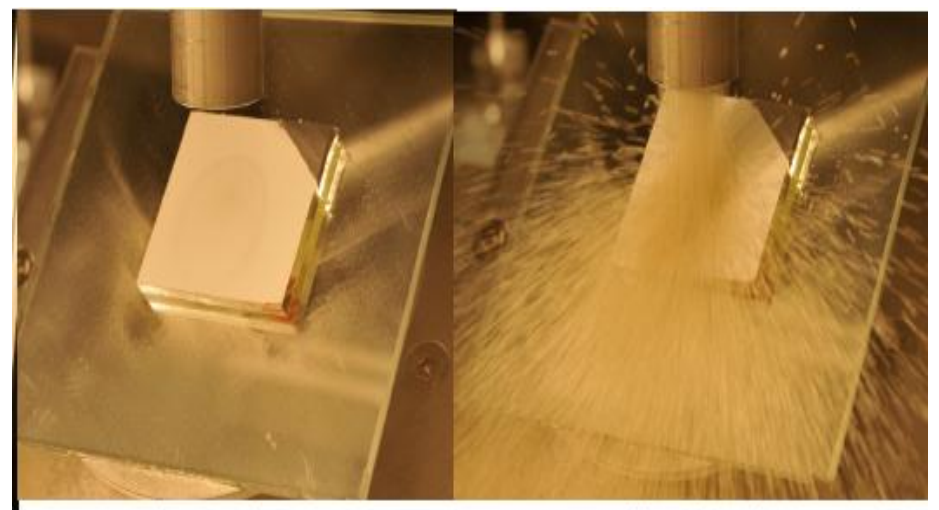

Integrated mirror probe in test section Running test, sand hits probe under $45^{\circ}$ angle

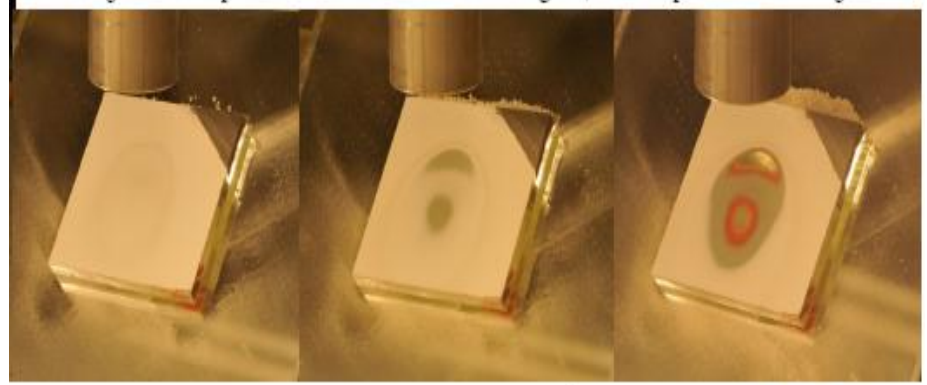

ent

Documentation of test surface damages

Fig -9: Sand Abrasion Test 


\subsubsection{Sand Abrasion Test on Front Side of the Mirror}

Table -1: Sand Abrasion Mirror Reading before test

\begin{tabular}{|l|l|l|l|l|l|}
\hline Time & $\begin{array}{l}\text { Altitude } \\
\text { Angle }\end{array}$ & $\begin{array}{l}\text { Azimuth } \\
\text { angle }\end{array}$ & $\begin{array}{l}\text { Global } \\
\text { radiation }\end{array}$ & $\begin{array}{l}\text { Diffused } \\
\text { radiation }\end{array}$ & $\begin{array}{l}\text { Reflected } \\
\text { radiation }\end{array}$ \\
\hline 1.22 & 77.57 & 273.23 & 748 & 158 & 324 \\
\hline 1.23 & 77.27 & 273.25 & 749 & 159 & 322 \\
\hline 1.24 & 76.96 & 273.31 & 752 & 162 & 315 \\
\hline 1.25 & 76.65 & 273.28 & 753 & 161 & 323 \\
\hline 1.26 & 76.35 & 273.30 & 753 & 162 & 321 \\
\hline 1.27 & 75.81 & 273.35 & 753 & 161 & 322 \\
\hline 1.28 & 75.63 & 273.37 & 746 & 162 & 315 \\
\hline 1.29 & 75.24 & 273.42 & 747 & 165 & 323 \\
\hline
\end{tabular}

Table -2: Sand Abrasion Mirror Reading after test

\begin{tabular}{|l|l|l|l|l|l|}
\hline Time & $\begin{array}{l}\text { Altitude } \\
\text { Angle }\end{array}$ & $\begin{array}{l}\text { Azimuth } \\
\text { angle }\end{array}$ & $\begin{array}{l}\text { Global } \\
\text { radiation }\end{array}$ & $\begin{array}{l}\text { Diffused } \\
\text { radiation }\end{array}$ & $\begin{array}{l}\text { Reflected } \\
\text { radiation }\end{array}$ \\
\hline 2.02 & 68.79 & 274.35 & 716 & 162 & 4 \\
\hline 2.03 & 68.72 & 274.38 & 715 & 160 & 4 \\
\hline 2.04 & 68.15 & 274.40 & 712 & 159 & 4 \\
\hline 2.05 & 67.98 & 274.42 & 710 & 157 & 5 \\
\hline 2.06 & 67.71 & 274.41 & 709 & 158 & 5 \\
\hline 2.07 & 67.47 & 274.40 & 708 & 156 & 5 \\
\hline 2.08 & 67.28 & 274.47 & 705 & 155 & 5 \\
\hline 2.09 & 66.89 & 274.53 & 699 & 152 & 5 \\
\hline
\end{tabular}

\subsection{Salt Water Spray}

2) The procedure is same for this test.

1) This test is similar to the sand abrasion test only difference is mixture of salt and water is used instead of sand.

\subsubsection{Salt Spray Test on the Mirror}

\subsubsection{Test on Front Side of the Mirror}

Table -3: Salt Spray Mirror Reading before test (Front side)

\begin{tabular}{|l|l|l|l|l|l|}
\hline Time & $\begin{array}{l}\text { Altitude } \\
\text { Angle }\end{array}$ & $\begin{array}{l}\text { Azimuth } \\
\text { angle }\end{array}$ & $\begin{array}{l}\text { Global } \\
\text { radiation }\end{array}$ & $\begin{array}{l}\text { Diffused } \\
\text { radiation }\end{array}$ & $\begin{array}{l}\text { Reflected } \\
\text { radiation }\end{array}$ \\
\hline 2.21 & 63.39 & 274.5 & 689 & 105 & 351 \\
\hline 2.22 & 62.90 & 274.61 & 691 & 101 & 358 \\
\hline 2.23 & 62.75 & 274.63 & 690 & 102 & 345 \\
\hline 2.24 & 62.52 & 274.67 & 689 & 103 & 350 \\
\hline 2.45 & 57.56 & 275.57 & 643 & 102 & 320 \\
\hline 2.46 & 57.17 & 275.64 & 646 & 100 & 320 \\
\hline 2.47 & 57.03 & 275.67 & 646 & 102 & 316 \\
\hline 2.48 & 56.85 & 275.70 & 644 & 99 & 307 \\
\hline
\end{tabular}


Table -4: Salt Spray Mirror Reading after test (Front side)

\begin{tabular}{|l|l|l|l|l|l|}
\hline Time & $\begin{array}{l}\text { Altitud } \\
\text { e Angle }\end{array}$ & $\begin{array}{l}\text { Azimuth } \\
\text { angle }\end{array}$ & $\begin{array}{l}\text { Global } \\
\text { radiation }\end{array}$ & $\begin{array}{l}\text { Diffused } \\
\text { radiation }\end{array}$ & $\begin{array}{l}\text { Reflected } \\
\text { radiation }\end{array}$ \\
\hline 11.56 & 82.64 & 86.91 & 761 & 154 & 65 \\
\hline 11.57 & 82.77 & 86.90 & 762 & 154 & 60 \\
\hline 11.58 & 82.93 & 86.89 & 765 & 154 & 51 \\
\hline 11.59 & 83.26 & 86.85 & 760 & 154 & 57 \\
\hline 12.00 & 83.38 & 86.83 & 757 & 155 & 62 \\
\hline 12.01 & 83.58 & 86.80 & 759 & 154 & 60 \\
\hline 12.09 & 85.51 & 86.21 & 780 & 157 & 66 \\
\hline 12.10 & 85.74 & 85.08 & 784 & 161 & 60 \\
\hline
\end{tabular}

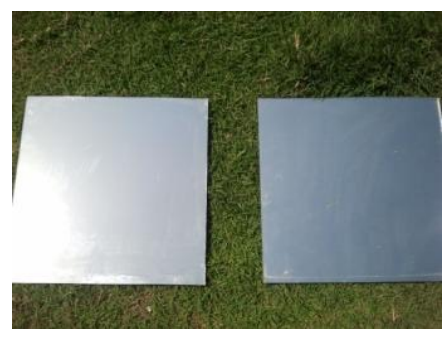

Fig -10: Before Test

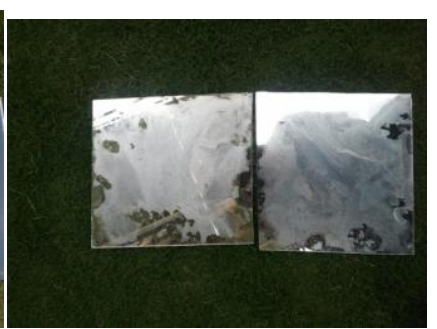

Fig -11: After Test

\section{Graphical Representation:}

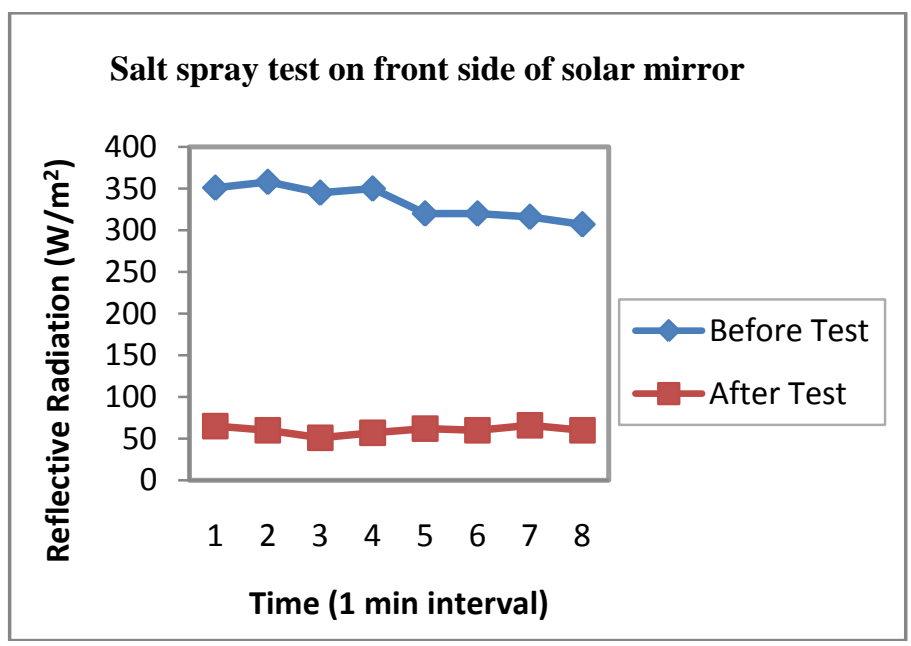

\subsubsection{Test on Rear Side of the Mirror}

Table -5: Salt Spray Mirror Reading before test (Rear side)

\begin{tabular}{|l|l|l|l|l|l|}
\hline Time & $\begin{array}{l}\text { Altitude } \\
\text { Angle }\end{array}$ & $\begin{array}{l}\text { Azimuth } \\
\text { angle }\end{array}$ & $\begin{array}{l}\text { Global } \\
\text { radiation }\end{array}$ & $\begin{array}{l}\text { Diffused } \\
\text { radiation }\end{array}$ & $\begin{array}{l}\text { Reflected } \\
\text { radiation }\end{array}$ \\
\hline 1.26 & 76.35 & 273.30 & 753 & 162 & 321 \\
\hline 1.27 & 75.81 & 273.35 & 753 & 161 & 322 \\
\hline 1.28 & 75.63 & 273.37 & 746 & 162 & 315 \\
\hline 1.29 & 75.24 & 273.42 & 747 & 165 & 323 \\
\hline 1.30 & 75.01 & 273.43 & 751 & 165 & 322 \\
\hline 1.31 & 74.73 & 273.47 & 750 & 167 & 324 \\
\hline 1.32 & 74.60 & 273.48 & 755 & 168 & 321 \\
\hline 1.33 & 74.35 & 273.51 & 757 & 171 & 327 \\
\hline 1.34 & 74.21 & 273.52 & 760 & 171 & 325 \\
\hline
\end{tabular}


Table -6: Salt Spray Mirror Reading after test (Rear side)

\begin{tabular}{|l|l|l|l|l|l|}
\hline Time & $\begin{array}{l}\text { Altitude } \\
\text { Angle }\end{array}$ & $\begin{array}{l}\text { Azimuth } \\
\text { angle }\end{array}$ & $\begin{array}{l}\text { Global } \\
\text { radiation }\end{array}$ & $\begin{array}{l}\text { Diffused } \\
\text { radiation }\end{array}$ & $\begin{array}{l}\text { Reflected } \\
\text { radiation }\end{array}$ \\
\hline 2.06 & 67.71 & 274.41 & 709 & 158 & 294 \\
\hline 2.07 & 67.47 & 274.44 & 708 & 156 & 291 \\
\hline 2.08 & 67.28 & 274.47 & 705 & 155 & 297 \\
\hline 2.09 & 66.89 & 274.53 & 699 & 152 & 296 \\
\hline 2.10 & 66.76 & 274.55 & 697 & 150 & 296 \\
\hline 2.11 & 66.43 & 274.60 & 691 & 146 & 300 \\
\hline 2.12 & 66.24 & 274.63 & 688 & 145 & 296 \\
\hline 2.13 & 66.01 & 274.67 & 691 & 144 & 302 \\
\hline 2.14 & 65.97 & 274.70 & 688 & 142 & 300 \\
\hline
\end{tabular}

\section{Graphical Representation:}

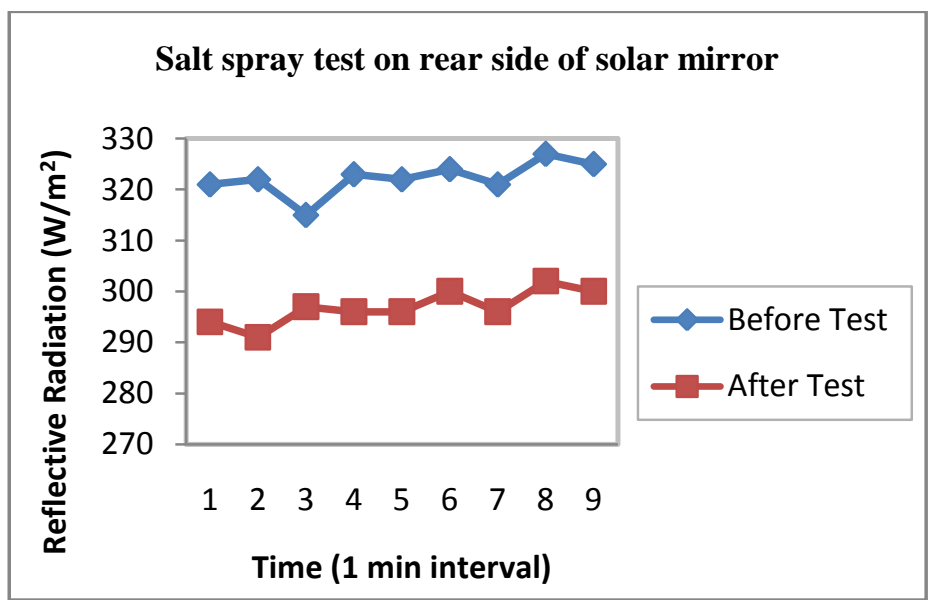

\section{RESULTS}

1) Incident radiation $=\left[\left(\mathrm{I}_{\mathrm{g}}-\mathrm{I}_{\mathrm{d}}\right) / \cos (90-\alpha)\right]$

2) Reflected Radiation $=\left(\rho^{2}\right) *($ Incident Radiation $)$ (Observed)

\subsection{Salt Spray Test on Front Side}

Table -7: Salt Spray Calculated Reading before test

\begin{tabular}{|l|l|l|l|l|l|}
\hline Time & $\begin{array}{l}\text { Global } \\
\text { Radiation }\end{array}$ & $\begin{array}{l}\text { Reflected } \\
\text { Radiation }\end{array}$ & $\begin{array}{l}\text { Altitude } \\
\text { Angle }\end{array}$ & $\begin{array}{l}\text { Incident } \\
\text { Radiation }\end{array}$ & Reflectivity \\
\hline 2.21 & 689 & 105 & 63.39 & 653.18 & 0.7333 \\
\hline 2.22 & 691 & 101 & 62.90 & 662.76 & 0.7350 \\
\hline 2.23 & 690 & 102 & 62.75 & 661.40 & 0.7222 \\
\hline 2.24 & 689 & 103 & 62.52 & 660.52 & 0.7279 \\
\hline 2.45 & 643 & 102 & 57.56 & 641.03 & 0.7065 \\
\hline 2.46 & 646 & 100 & 57.17 & 649.78 & 0.7017 \\
\hline 2.47 & 646 & 102 & 57.03 & 648.42 & 0.6980 \\
\hline 2.48 & 644 & 99 & 56.85 & 650.94 & 0.6860 \\
\hline
\end{tabular}


Table -8: Salt Spray Calculated Reading after test

\begin{tabular}{|l|l|l|l|l|l|}
\hline Time & $\begin{array}{l}\text { Global } \\
\text { Radiation }\end{array}$ & $\begin{array}{l}\text { Reflected } \\
\text { Radiation }\end{array}$ & $\begin{array}{l}\text { Altitude } \\
\text { Angle }\end{array}$ & $\begin{array}{l}\text { Incident } \\
\text { Radiation }\end{array}$ & Reflectivity \\
\hline 11.56 & 761 & 65 & 82.64 & 612.05 & 0.3258 \\
\hline 11.57 & 762 & 60 & 82.77 & 612.87 & 0.3128 \\
\hline 11.58 & 765 & 51 & 82.93 & 615.68 & 0.2878 \\
\hline 11.59 & 760 & 57 & 83.26 & 610.21 & 0.3656 \\
\hline 12.00 & 757 & 62 & 83.38 & 606.04 & 0.3196 \\
\hline 12.01 & 759 & 60 & 83.58 & 608.82 & 0.3139 \\
\hline 12.09 & 780 & 66 & 85.51 & 624.92 & 0.3249 \\
\hline 12.10 & 784 & 60 & 85.74 & 624.72 & 0.3099 \\
\hline
\end{tabular}

Percentage decrease in reflectivity on front side $=56.22 \%$

\subsection{Salt Spray Test on Rear Side}

Table -9: Salt Spray Calculated Reading before test

\begin{tabular}{|l|l|l|l|l|l|}
\hline Time & $\begin{array}{l}\text { Global } \\
\text { Radiation }\end{array}$ & $\begin{array}{l}\text { Reflected } \\
\text { Radiation }\end{array}$ & $\begin{array}{l}\text { Altitude } \\
\text { Angle }\end{array}$ & $\begin{array}{l}\text { Incident } \\
\text { Radiation }\end{array}$ & Reflectivity \\
\hline 1.26 & 753 & 321 & 76.35 & 608.17 & 0.7265 \\
\hline 1.27 & 753 & 322 & 75.81 & 610.63 & 0.7261 \\
\hline 1.28 & 746 & 315 & 75.63 & 602.86 & 0.7228 \\
\hline 1.29 & 747 & 323 & 75.24 & 601.86 & 0.7325 \\
\hline 1.30 & 751 & 322 & 75.01 & 606.64 & 0.7285 \\
\hline 1.31 & 750 & 324 & 74.73 & 604.33 & 0.7322 \\
\hline 1.32 & 755 & 321 & 74.60 & 608.86 & 0.7260 \\
\hline 1.33 & 757 & 327 & 74.35 & 608.56 & 0.7330 \\
\hline 1.34 & 760 & 325 & 74.21 & 612.10 & 0.7287 \\
\hline
\end{tabular}

Table -10: Salt Spray Calculated Reading after test

\begin{tabular}{|l|l|l|l|l|l|}
\hline Time & $\begin{array}{l}\text { Global } \\
\text { Radiation }\end{array}$ & $\begin{array}{l}\text { Reflected } \\
\text { Radiation }\end{array}$ & $\begin{array}{l}\text { Altitude } \\
\text { Angle }\end{array}$ & $\begin{array}{l}\text { Incident } \\
\text { Radiation }\end{array}$ & Reflectivity \\
\hline 2.06 & 709 & 294 & 67.71 & 595.49 & 0.7013 \\
\hline 2.07 & 708 & 291 & 67.47 & 597.61 & 0.6986 \\
\hline 2.08 & 705 & 297 & 67.28 & 596.26 & 0.7057 \\
\hline 2.09 & 699 & 296 & 66.89 & 594.72 & 0.7054 \\
\hline 2.10 & 697 & 296 & 66.76 & 595.30 & 0.7051 \\
\hline 2.11 & 691 & 300 & 66.43 & 594.60 & 0.7103 \\
\hline 2.12 & 688 & 296 & 66.24 & 593.28 & 0.7063 \\
\hline 2.13 & 691 & 302 & 66.01 & 598.72 & 0.7102 \\
\hline 2.14 & 688 & 300 & 65.97 & 597.81 & 0.7084 \\
\hline
\end{tabular}

Percentage decrease in reflectivity on rear side $=1.9 \%$ 


\subsection{Sand Abrasion Test on Front Side}

Table -11: Sand Abrasion Calculated Reading before test

\begin{tabular}{|l|l|l|l|l|l|}
\hline Time & $\begin{array}{l}\text { Global } \\
\text { Radiation }\end{array}$ & $\begin{array}{l}\text { Reflected } \\
\text { Radiation }\end{array}$ & $\begin{array}{l}\text { Altitude } \\
\text { Angle }\end{array}$ & $\begin{array}{l}\text { Incident } \\
\text { Radiation }\end{array}$ & Reflectivity \\
\hline 1.22 & 748 & 324 & 77.57 & 604.16 & 0.7323 \\
\hline 1.23 & 749 & 322 & 77.27 & 604.86 & 0.7296 \\
\hline 1.24 & 752 & 315 & 76.96 & 605.61 & 0.7212 \\
\hline 1.25 & 753 & 323 & 76.65 & 601.44 & 0.7286 \\
\hline 1.26 & 753 & 321 & 76.35 & 608.17 & 0.7265 \\
\hline 1.27 & 753 & 322 & 75.81 & 610.63 & 0.7261 \\
\hline 1.28 & 746 & 315 & 75.63 & 602.86 & 0.7228 \\
\hline 1.29 & 747 & 323 & 75.24 & 601.86 & 0.7325 \\
\hline
\end{tabular}

Table -12: Sand Abrasion Calculated Reading after test

\begin{tabular}{|l|l|l|l|l|l|}
\hline Time & $\begin{array}{l}\text { Global } \\
\text { Radiation }\end{array}$ & $\begin{array}{l}\text { Reflected } \\
\text { Radiation }\end{array}$ & $\begin{array}{l}\text { Altitude } \\
\text { Angle }\end{array}$ & $\begin{array}{l}\text { Incident } \\
\text { Radiation }\end{array}$ & Reflectivity \\
\hline 2.02 & 716 & 4 & 68.79 & 594.25 & 0.0820 \\
\hline 2.03 & 715 & 4 & 68.72 & 597.86 & 0.0818 \\
\hline 2.04 & 712 & 4 & 68.15 & 595.80 & 0.0819 \\
\hline 2.05 & 710 & 5 & 67.98 & 596.51 & 0.0915 \\
\hline 2.06 & 709 & 5 & 67.71 & 595.49 & 0.0916 \\
\hline 2.07 & 708 & 5 & 67.47 & 593.61 & 0.0914 \\
\hline 2.08 & 705 & 5 & 67.28 & 596.26 & 0.0915 \\
\hline 2.09 & 699 & 5 & 66.89 & 594.72 & 0.0917 \\
\hline
\end{tabular}

Percentage decrease in reflectivity on front side $=87.92 \%$

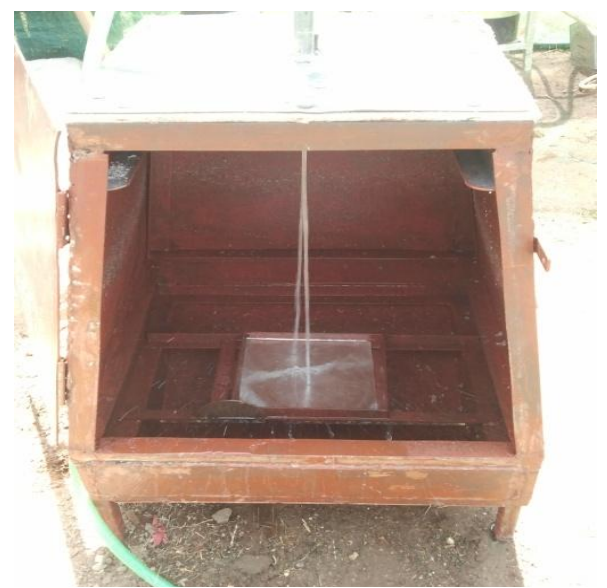

Fig -12: Mirror in Testing Process

\section{CONCLUSIONS}

The various test conditions required were water, salt, sand and temperature effects. When the chamber was manufactured, salt spray test was conducted on both the sides of the solar mirror. The reflectivity of the unused mirrors was first obtained from the reflectivity setup. Then the same mirrors were subjected to the salt spray and the readings were observed. From the observed readings it is clear that the amount of reflected radiation decreased by the test conducted on the solar mirror. The results obtained were far more different from the expected values. The reflected radiation was almost half of the initial value at some points. Hence the test showed the actual variation in the radiations which is helpful in designing the mirrors with specific properties that are resistant to such condition. Even if the upgrade to these special mirrors is not possible, one can prepare a maintenance schedule based on the results. This will ensure high reflectivity of the solar mirrors during their operation.

\section{ACKNOWLEDGMENTS}

The author would like to thank the anonymous reviewers for their comments which were very helpful in improving the quality and presentation of this paper. 


\section{REFERENCES}

[1] Daly, J.C., 1979. Solar concentrator flux distributions using backward ray tracing. Applied Optics 18 (15), 2696-2699.

[2] Hutchinson, T.P., Lai, C.D., 1991. The Engineering Statistician's Guide to continuous Bivariate Distributions. Rumsby Scientific Publishing, pp. 280.

[3] Jeter, S.M., 1986. The distribution of concentrated solar radiation in paraboloidal collectors. Journal of Solar Energy Engineering 108, 219-225.

[4] Johnston, G., 1995. On the analysis of surface error distributions on concentrated solar collectors. Journal of Solar Energy Engineering 117 (4), 294-296.

[5] Ronnen Levinson, Hashem Akbari, and Paul Berdahl. Measuring solar reflectance Part I: defining a metric that accurately predicts solar heat gain.

[6] Charlie Moore. The solar spectrum reectometer (SSR). Technical Note 79-16, Devices \& Services, Dallas, TX, 1979.

[7] http://www.sciencedirect.com/science/article/pii/0038092 X59900039

[8] C.F. Chen, C.H. Lin, H.T.Jan \& Y.L. Yang, “ Design of a Solar Concentrator Combining Paraboloidal and Hyperbolic Mirrors Using Ray Tracing Method", Optics Communication, 282: 360-366, 2009.

[9] V.B. Omubo-Pepple, C. Israel-Cookey and G.I. Alaminokuma, " Effects of Temperature, Solar Flux and Relative Humidity on the Efficient Conversion of Solar Energy to Electricity", European Journal of Scientific Research, 35(2): 173-180, 2009. 